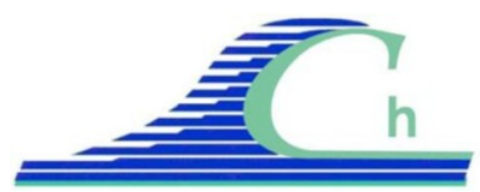

XII ${ }^{\text {ìmes }}$ Journées Nationales Génie Côtier - Génie Civil

Cherbourg, 12-14 juin 2012

DOI:10.5150/jngcgc.2012.012-M C Editions Paralia CFL

disponible en ligne - http://www.paralia.fr - available online

\title{
Lien entre la forme locale de la surface libre et les pressions d'impact générées par une vague déferlante sur un ouvrage
}

\author{
Cyril MOKRANI $^{1,2}$, Stéphane ABADIE ${ }^{1}$, Kamel ZIBOUCHE ${ }^{2}$
}

1. Univ. Pau \& Pays Adour, SIAME - EA 4581 - Laboratoire des Sciences de l’Ingénieur Appliquées à la Mécanique et au Génie Électrique, Allée du Parc Montaury, Anglet, 64600, France. cyril.mokrani@univ-pau.fr; stephane.abadie@univ-pau.fr

2. Centre Scientifique et Technique du Bâtiment. 84, avenue Jean Jaurès, Champs-sur-Marne, 77447 Marne-la-Vallée Cédex 2, France.

kamel.zibouche@cstb.fr

\section{Résumé :}

Des impacts de vagues déferlantes sur un obstacle vertical ont été simulés à l'aide d'un modèle Navier-Stokes avec suivi d'interface VOF. Les résultats montrent que les pics de pression obtenus sont extrêmement variables et que cette variabilité est due à la forme locale de l'interface avant impact. En se ramenant au cas du jet triangulaire, nous expliquons pourquoi il en est ainsi et montrons que le code peut néanmoins estimer assez précisément les pics de pression à condition de connaître précisément la forme locale de l'interface avant impact. Dans ces conditions, le modèle apparaît fiable tant que l'angle entre l'interface eau/air et l'ouvrage est supérieur à $30^{\circ}$. Lorsque cet angle se rapproche de 0 , l'impact met en jeu des vitesses verticales et des pressions associées pour lesquelles l'erreur des résultats numériques devient importante.

\section{Mots-clés :}

Déferlement - Ouvrage - Pression d'impact - Efforts de percussion - Simulation

\section{Introduction}

L'interaction d'une vague avec une structure verticale génère sur cette dernière des pressions locales qui varient en temps et en espace. Différentes études ont montré que le signal de pression en un point fixe dépend fortement des caractéristiques de la vague incidente. Lorsque l'impact met en jeu des vagues de fortes cambrures ou des vagues déferlantes, les efforts sont très intenses et apparaissent sur un intervalle de temps très court. Dans ce cas, on parle d'impacts percussifs. Les sollicitations générées sur la structure par ce type d'effort sont assez mal connues et nécessitent des études de recherche visant à les intégrer de manière plus précise dans le dimensionnement des ouvrages côtiers. Les différentes études expérimentales consacrées à ce sujet montrent que des valeurs très différentes de pression sont en général obtenues pour des forçages similaires (e.g., WITTE, 1988). Les auteurs attribuent cette forte variabilité des mesures aux conditions locales d'impact (i.e. forme de la surface libre) ainsi qu'au rôle de l'air et 
de sa compressibilité associée (air piégé, e.g. LUGNI et al., 2006, ou air entraîné, e.g., PLUMERAULT, 2009). Il est néanmoins difficile de déconnecter ces deux paramètres forme de l'interface et quantité air - expérimentalement. Au contraire, la simulation numérique apparaît plus adaptée pour une étude fine de chaque processus. Elle apporte également plus de souplesse que l'expérimentation physique. Cependant, les différentes tentatives visant à estimer numériquement les pressions d'impacts ont soulevées des problèmes qui restent non résolus à l'heure actuelle : ARAI et al. (2002) utilisent un code 1 fluide et montrent que les pics de pressions calculés pendant un impact dépendent de la taille des mailles ainsi qu'aux pas de temps utilisés lors des simulations. KLEEFMAN et al. (2005) utilisent une méthode VOF couplée Level-Set et obtiennent des pics de pression instables et non réalistes. CHEN et al. (2009) utilisent un code RANS et obtiennent des signaux de pression qui dépendent de l'épaisseur de l'interface. Dans cet article, nous proposons d'étudier les capacités d'un code à calculer les pics de pression lors d'impacts percussifs puis de relier les valeurs de pression obtenues à la forme locale de l'interface eau / air avant impact.

\section{Description du modèle}

Le modèle THETIS (e.g., ABADIE et al., 2010) utilisé pour ces simulations résout les équations de Navier-Stokes incompressible dans un domaine composé d'eau et d'air par une formulation 1-fluide (e.g., ABADIE, 1998) dont les propriétés physiques (i.e., masse volumique et viscosité) dépendent du point. Les équations du mouvement sont discrétisées par la méthode des volumes finis sur un maillage fixe. A chaque itération, les champs des vitesses et de pression sont obtenus en tout point du maillage par la méthode du Lagrangien Augmenté (LUBIN, 2004). Le mouvement de l'interface eau / air est ensuite résolu à l'aide de la méthode VOF de type CIAM rendue légèrement diffuse sur 2 mailles (MOKRANI, 2012) pour éviter des problèmes d'instabilités numériques. La connaissance de la position de l'interface permet ensuite de recalculer les nouvelles valeurs de densité et viscosité locales par une simple interpolation basée sur la fraction volumique. Les obstacles sont introduits dans le domaine de calcul par une méthode de pénalisation (LUBIN, 2004).

\section{Cas du jet triangulaire}

Afin d'étudier les capacités du code à estimer les pics de pression lors d'un impact percussif, nous avons dans un premier temps étudié le cas académique du jet triangulaire (figure 1). Il s'agit d'une représentation simplifiée de l'impact d'une vague au niveau local. Lorsque l'interface rencontre l'obstacle, les forces de pression locales au niveau de la paroi forcent l'écoulement à évoluer. L'endroit de moindre résistance étant la surface libre au voisinage de l'impact $(\mathrm{P}=0)$, un jet ascendant se forme dans cette zone conformément au schéma de la figure 1. Sur cette figure, on a dessiné en pointillé la position de l'interface à t=dt comme s'il n'y avait pas de paroi. 


\section{XII ${ }^{\text {èmes }}$ Journées Nationales Génie Côtier - Génie Civil \\ Cherbourg, 12-14 juin 2012}

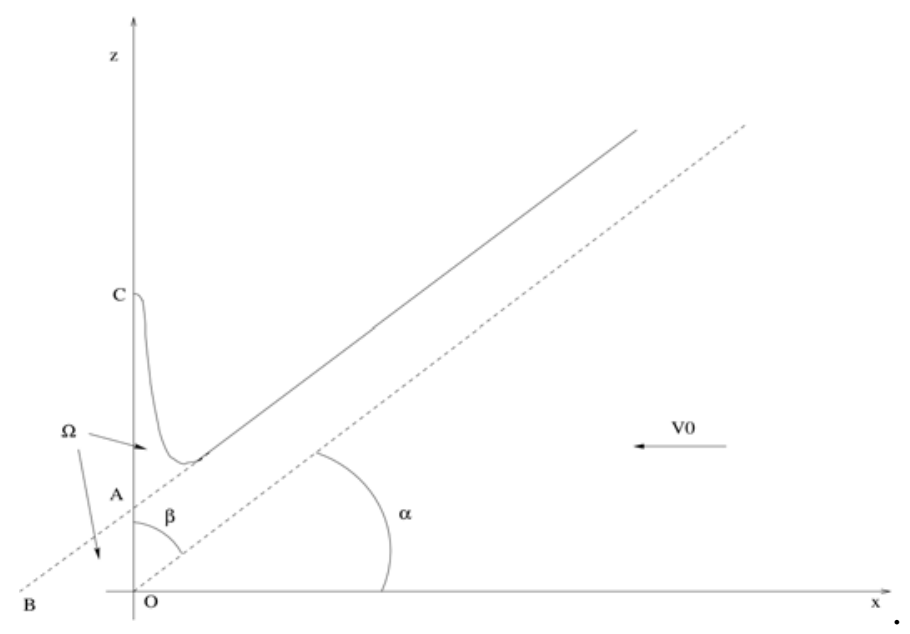

Figure 1. Schématisation de l'impact d'un jet triangulaire sur une paroi. En trait pointillé : interface avant impact $(t=0)$. En trait continu : interface déformée à $t=d t$.

Une simple conservation du volume permet d'observer que le volume $\Omega$ du jet ascendant se calcule facilement par l'aire du triangle OAB. Étant donné que le jet avance à vitesse constante $\mathrm{V}_{0}$, on $\mathrm{a}: \mathrm{OB}=\mathrm{V}_{0}$ dt et $\mathrm{OA}=\mathrm{V}_{\mathrm{O}} \mathrm{dt} \tan (\alpha)$, d'où $\Omega=\left(\mathrm{V}_{0} \mathrm{dt}\right)^{2} \tan (\alpha)$. On remarque également que la vitesse verticale du point géométrique A est : $V_{A}=V_{0} \tan (\alpha)$. Ainsi, plus l'angle $\alpha$ est proche de $\pi / 2$ (ou $\beta$ proche de 0 ), plus le volume à évacuer en un temps dt est grand (il n'est d'ailleurs pas borné). De même, la vitesse verticale du point géométrique $\mathrm{A}$ augmente aussi très fortement (en étant également non bornée) plus $\alpha$ se rapproche de $\pi / 2$. Entre $t=0$ et $t=d t$, les particules fluides sur la paroi passent d'une vitesse verticale nulle à une vitesse de l'ordre de $\mathrm{V}_{\mathrm{A}}$. L'équation de Navier-Stokes projetée sur la verticale s'écrit (avec la vitesse normale à la paroi $\mathrm{U}=0$ et en négligeant la gravité) :

$$
\rho \frac{D V}{D t}=-\frac{\partial P}{\partial z}
$$

Localement donc, l'accélération de la particule (pour passer de 0 à $V_{A}$ ) génère un gradient vertical de pression. Ce gradient de pression (et donc indirectement la pression d'impact) sera d'autant plus fort que la borne à atteindre $\left(\mathrm{V}_{\mathrm{A}}\right)$ est éloigné de 0 . Ce raisonnement simple permet ainsi de saisir le lien entre l'angle d'incidence locale de l'interface et l'intensité de la pression d'impact intimement liée à la vitesse verticale dans le jet. Il permet aussi de mieux saisir intuitivement pourquoi des variations faibles de l'angle d'incidence de la surface libre peuvent induire de larges variations de pression et de vitesse.

Par ailleurs, les solutions de ce problème sans grandeurs géométriques caractéristiques sont auto-similaires ce qui signifie que les évolutions en temps des variables (pression, surface libre, etc) sont homothétiques de rapport $V_{0} t$. WU (2007) résout cet écoulement en théorie potentielle par la technique des éléments frontières et valide son modèle par 
rapport à d'autres solutions obtenues en partant de l'hypothèse d'auto-similarité. Il obtient respectivement des pressions maximales (indépendante du temps de par l'autosimilarité) $\mathrm{P}_{\max } / \mathrm{P}_{0}$ où $\mathrm{P}_{0}=\rho \mathrm{V}_{0}^{2}$ de $\sim 2,4.5,40$ pour des angles $\alpha$ valant respectivement $45^{\circ}, 60^{\circ}$ et $80^{\circ}$ (soit des angles $\beta$ entre la surface libre et la paroi de $45^{\circ}, 30^{\circ}$ et $10^{\circ}$ ). La forte disparité des valeurs souligne l'importance de la connaissance fine de cet angle local avant l'impact.
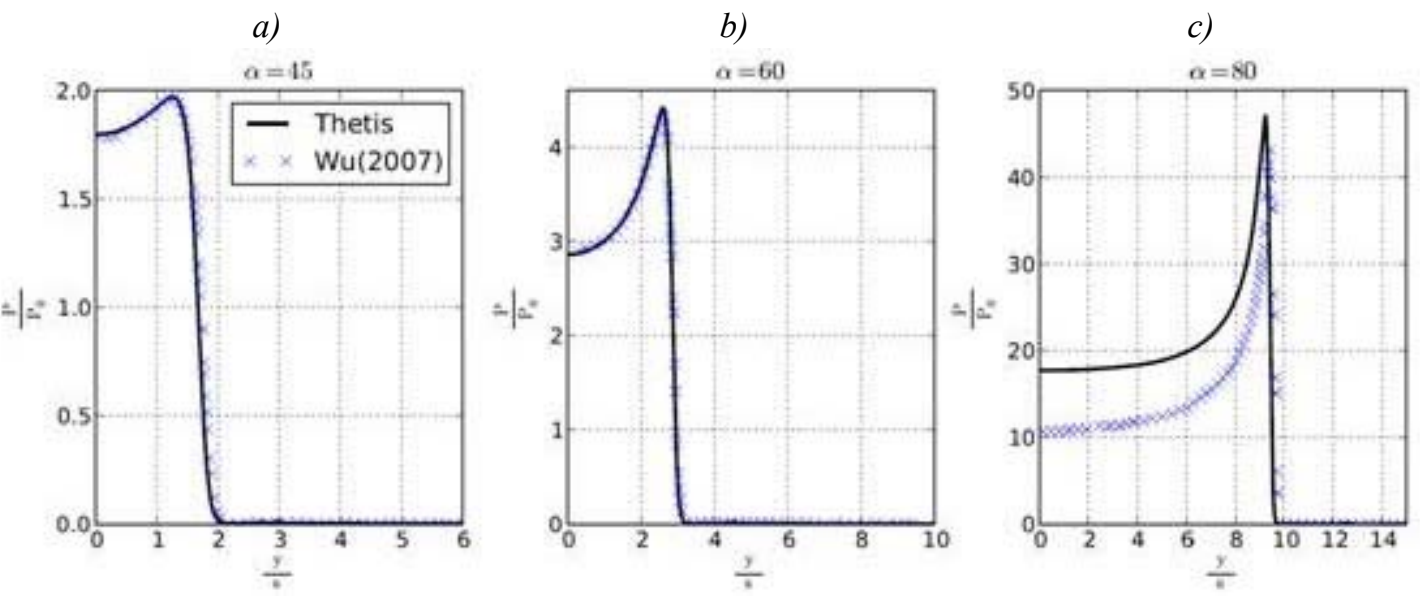

Figure 2. Évolution de la pression le long de la paroi (y est l'altitude et $s=V_{0} t$ est le rapport d'homothétie) pour différents angles $\alpha$.

La question est maintenant de savoir si le modèle Navier-Stokes est capable ou non de reproduire ce type de phénomène. La réponse est oui mais avec une bonne précision et seulement sur une gamme limitée d'angle d'incidence. La figure 2 montre ainsi la comparaison des résultats numériques de THETIS avec ceux de WU (2007). Jusqu'à $\alpha=60^{\circ}$, les résultats concordent bien. Au-delà, des résultats satisfaisants sont obtenus mais ils sont à prendre avec précaution. La figure 2c permet déjà d'observer des différences entre les deux types de calculs. MOKRANI (2012) montre de plus qu'audessus de $\alpha=60-70^{\circ}$ (i.e. $\beta=40-30^{\circ}$ ), les calculs Navier-Stokes VOF ne respectent plus l'auto-similarité des solutions. Cela signifie que les variables de l'écoulement ne sont plus constantes dans les coordonnées autosimilaires $(\mathrm{x} / \mathrm{s}, \mathrm{y} / \mathrm{s})$ où $\mathrm{s}=\mathrm{V}_{0} \mathrm{t}$ ce qui n'est pas conforme à la théorie. En pratique, cela signifie donc aussi que le graphe en trait continu de la figure 2c n'est pas unique et il est donc difficile de le comparer aux résultats de WU (2007) qui eux le sont. Le code peu donc estimer les pics de pression pour des angles d'incidences inférieurs à $\alpha=60^{\circ}-70^{\circ}$. Dans ces conditions, $\alpha$ semble déterminer le signal de pression et par conséquent le pic de pression. 


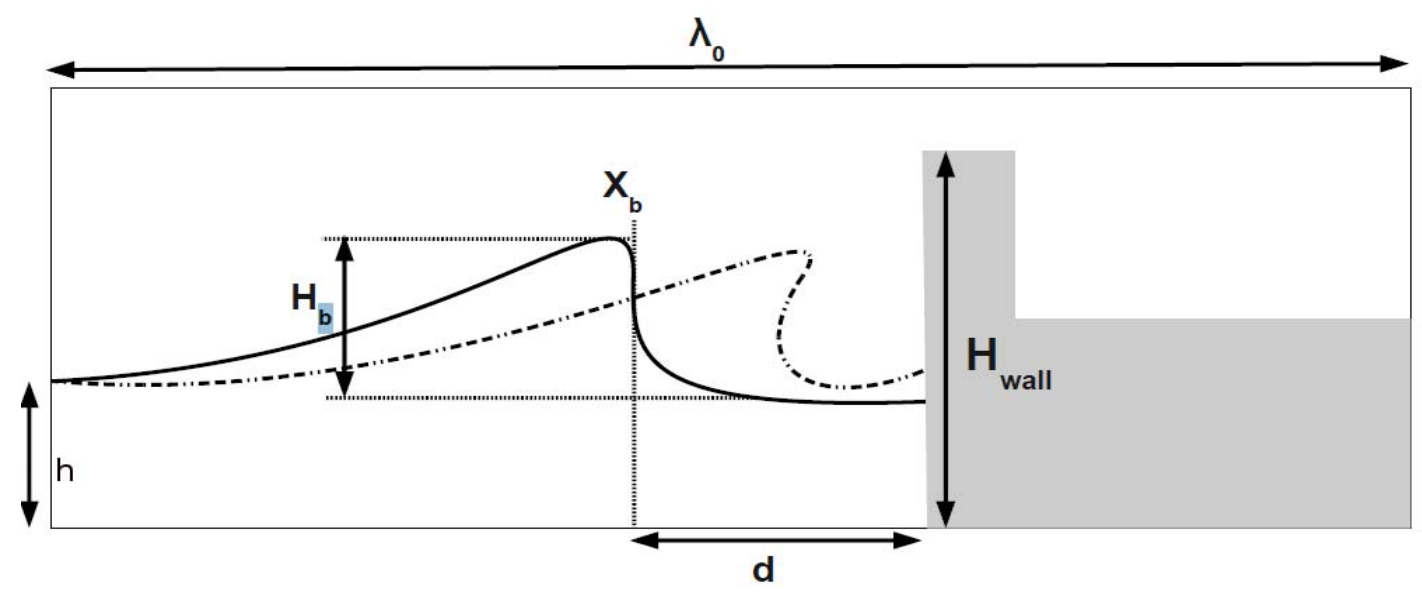

Figure 3. Impact d'une onde de Stokes instable sur un ouvrage vertical à position horizontale variable. Définition de la configuration d'étude et paramètres associés.

\section{Illustration des problèmes liés au calcul numérique de pressions d'impact}

Revenons maintenant à un cas d'impact de vague déferlante et aux problèmes liés à l'estimation de pics de pression lors d'une percussion. On considère le problème schématisé sur la figure 3 . Pour ces simulations, le déferlement est obtenu à l'aide d'une onde de Stokes d'ordre 1 à forte amplitude initiale donc instable. Pendant cette phase de déferlement, l'obstacle visible sur la figure 3 n'est en fait pas présent. Il est "ajouté" dans la simulation au moment et à l'endroit souhaité. Bien que cette méthode ne rende pas compte des effets du ressac sur la forme de la surface libre, pourtant mise en évidence expérimentalement, elle permet d'analyser très simplement la capacité du code à calculer les pressions d'impact dans différents cas d'impact (avec / sans air piégé, forte / faible cambrure etc.) et de mettre ainsi en évidence les capacités du modèle à reproduire des pics de pression. Ajoutons enfin que la compressibilité de l'air n'étant pas pris en compte dans ces simulations, les valeurs numériques obtenues après l'impact doivent être pris avec précaution.

En faisant varier légèrement la position de l'obstacle $d^{*}=d / h$ vis à vis du point de déferlement, les conditions d'impact sont modifiés. Les résultats de ces simulations sont présentés sur la figure 4a. Chaque courbe représente le maximum de pression obtenu sur l'ouvrage en fonction du temps pour un maillage $\Delta \mathrm{x}^{*}=\Delta \mathrm{z}^{*}=3.84 .10^{-2}$ (où $\Delta \mathrm{x}^{*}=\Delta \mathrm{x} / \mathrm{h}$ ). La différence entre les courbes est due aux différentes valeurs de $\mathrm{d}^{*}$ considérées (indiquées sur le graphe). Il semble à première vue et dans ce cas précis, qu'une variation de position de l'ouvrage de l'ordre de la profondeur par rapport au point de déferlement entraîne une augmentation du pic de pression de l'ordre de 50\%. 
a)

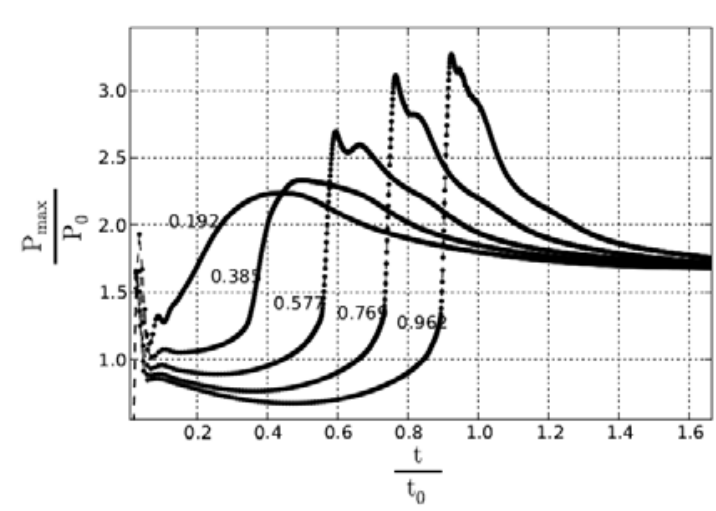

b)

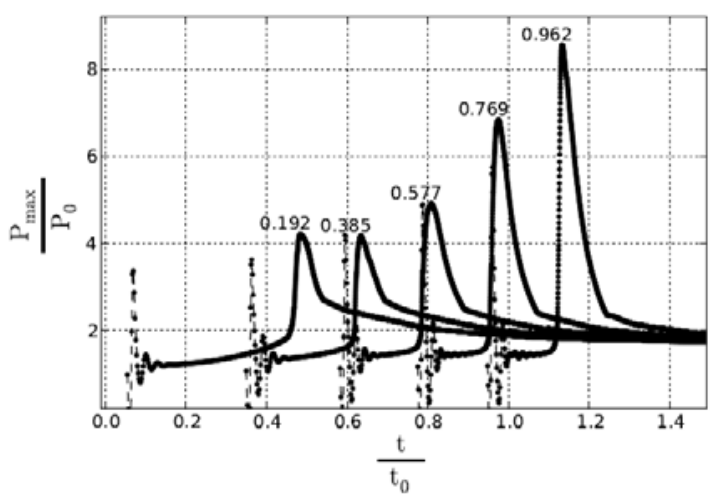

Figure 4. Valeurs maximales de la pression adimensionnelle $\left(P_{0}=\rho c_{0}{ }^{2}\right.$ où $c_{0}$ est la célérité théorique de l'onde) sur l'ouvrage en fonction du temps adimensionnel $\left(t_{0}=h / c_{0}\right)$ pour différentes position de l'ouvrage vis à vis du point de déferlement. a) pas d'espace $\Delta x^{*}=\Delta z^{*}=3.84 \times 10^{-2}$, b) maillage fin $\Delta x^{*}=\Delta z^{*}=9.61 \times 10^{-3}$.

Pour vérifier ce résultat, il convient néanmoins de montrer que les résultats numériques convergent lorsque l'espacement des mailles tend vers 0 , quelle que soit la valeur de $\mathrm{d}^{*}$. En réalisant des simulations identiques avec un pas d'espace quatre fois plus fin, nous obtenons les résultats de la figure $4 \mathrm{~b}$. Les pics de pression ont toujours tendance à augmenter avec la distance déferlement/ouvrage mais les valeurs des pics obtenus sont bien plus importantes (e.g., $8 \mathrm{P}_{0}$ contre $3.5 \mathrm{P}_{0}$ pour le cas $\mathrm{d}^{*}=0.96$ ). Au vue de ces résultats, nous avons refait la même expérience en fixant $\mathrm{d}^{*}$ à $\mathrm{d}^{*}=1,538$ et en utilisant différents maillages. Les résultats montrent que les valeurs maximales de pression ne se stabilisent pas et au contraire en extrapolant la tendance (RICHARDSON, 1911), on montre qu'elles divergent lorsque la taille des mailles est réduite, ce qui empêche toute estimation des pics de pression.

En fait, ce qui se passe est relativement simple mais a des implications importantes. Sur la figure 4a, on imagine bien que des différences au niveau de la géométrie de l'interface et du champ des vitesses expliquent les variations observées sur les pressions calculées. Il est extrêmement difficile d'assurer pour des simulations du même problème sur des maillages différents que les interfaces locales au moment de l'impact aient exactement les mêmes géométries et vitesses dans chaque cas. Or le phénomène est tellement sensible que le changement de maillage entrâne une instabilité des résultats. MOKRANI (2012) a également observé ce comportement instable des pressions dans le cas de l'impact d'une rupture de barrage sur une paroi verticale. MOKRANI (2012) montre ainsi que le raffinement du maillage entraîne un raidissement graduel de l'interface locale avant impact qui se traduit par des pressions qui augmentent constamment. Un des points intéressant que pose cette observation et pour lequel nous n'avons pas encore de réponse est pourquoi ce comportement est monotone, c'est à dire 


\section{XII ${ }^{\text {èmes }}$ Journées Nationales Génie Côtier - Génie Civil \\ Cherbourg, 12-14 juin 2012}

pourquoi les interfaces sont-elles toujours plus raides pour des maillages plus fins ? De plus, ce comportement divergent est observé sur un cas pour lequel l'air piégé n'est pas présent. La non prise en compte de la compressibilité n'est donc pas responsable du comportement divergent des pics de pression.

Une des implications de ces simulations est que de par la très forte sensibilité des pressions d'impact par rapport à la géométrie locale de l'interface, changer la taille des mailles revient à changer la nature même de l'impact, et par conséquent, revient à changer les pressions d'impact sur l'ouvrage. Il apparaît donc illusoire de tenter d'estimer des pressions sur ouvrage en simulant l'ensemble du processus depuis la levée jusqu'à l'impact car le filtre de l'impact amplifiera à l'extrême les moindres approximations numériques. La seule approche possible consiste à fixer des conditions locales d'impact parfaitement connues pour espérer avoir des valeurs d'effort réalistes.

\section{Conclusion}

Le cas du jet triangulaire permet de fixer une référence théorique simple qui aide à comprendre que les problèmes liés à l'estimation des pics de pression peuvent être dus à des changements de géométrie locale du profil d'interface pendant la phase qui précède l'impact. La principale conséquence de nos résultats est qu'il apparaît illusoire de simuler l'ensemble du mouvement (i.e., de la levée à l'impact) pour calculer des efforts sur ouvrage. Seule une forme de surface libre donnée avant impact permet d'obtenir un résultat unique. Ces différents résultats doivent cependant être nuancés : (i) le cas académique du jet triangulaire ne prend pas en compte la courbure du jet plongeant, les changements de vitesse du jet ainsi que l'inclinaison de la vitesse incidente du jet. YETTOU et al. (2007) montrent que les variations de vitesse incidentes ont une faible influence sur les valeurs maximales de pression lors d'un impact, cependant, l'influence des deux autres paramètres devrait être étudiée. (ii) Le comportement divergent des pics semble être dû à l'influence du maillage sur la forme de l'interface. Cependant, nous avons également mis en évidence les limites du code pour $\beta<30^{\circ}$. Ce dernier résultat n'est pas expliqué à l'heure actuelle mais nous pensons que ce problème vient de la méthode VOF. Cela montre que la forme locale de l'interface ne peut pas expliquer à elle seule tous les problèmes liés aux calculs des pics de pression. (iii) Tous les résultats ont été obtenus en utilisant un code NS-VOF. Le comportement des pics de pression mis en évidence dans cette étude est rigoureusement le même que celui décrit par ARAI et al. (2002) qui utilise un code 1 fluide avec une méthode des différences finies. En ce sens, le comportement des pics de pression obtenu pour des angles $\beta>30^{\circ}$ ne semble pas être du aux schémas numériques ou à la méthode de résolution des équations de NavierStokes, mais simplement à une mauvaise représentation de la géométrie du profil d'interface avant l'impact. 


\section{Références bibliographiques}

ABADIE S. (1998). Modélisation numérique du déferlement plongeant par méthode $V O F$. Thèse de doctorat, Université de Bordeaux I.

ABADIE S., MORICHON D., GRILLI S., GLOKNER S. (2010). Numerical simulation of waves generated by landslides using a multiple-fluid Navier-Stokes model. Coastal engineering, 57, pp 779-794. doi:10.1016/j.coastaleng.2010.03.003

ARAI M., CHENG L., KUNAMO A., MIYAMOTO T. (2002). A technique for stable numerical computaTion of hydrodynamic impact pressure in sloshing simulation. Journal of the society of naval architects of Japan 191, pp 299-307.

CHEN Y., DJIDJELI K., PRICE W. (2009). Numerical simulation of liquid sloshing phenomena in partially filled containers. Computers \& fluids 38, pp 830-842.

LUBIN P. (2004). Simulation des Grandes Echelles appliquée au déferlement plongeant des vagues. Université Bordeaux I.

LUGNI C., MIOZZI M., BROCCHINI M., FALTINSEN O.M. (2010). Evolution of the air cavity during a depressurized wave impact. I. The Kinematic Flow Field. Physics of fluids, Vol. 22, 056101.

KLEEFMAN K., FEKKEN, G., VELDMAN A., IWANOSKI B., BUCHNER B. (2005). A volume-of-fluid based simulation method for wave impact problems. Journal of computational physics, Vol. 206, pp 363-393.

MOKRANI C. (2012). Impacts de vagues déferlantes sur un obstacle vertical. Modèle théorique et estimation numérique des pics de pression. Thèse de doctorat, Université de Pau et des Pays de l'Adour.

PLUMERAULT L-R. (2009). Modélisation numérique d'impact de vagues sur un mur prise en compte de la présence d'air dans l'eau. Thèse de doctorat, Université de Pau et des Pays de l'Adour.

RICHARDSON L.F. (1911). The approximate arithmetical solution by finite differences of physical problems including differential equations with an application to the stresses in a masonry dam. Philo Trans of Royal Soc of London, Series A, 210, pp 307-357. doi:10.1098/rsta.1911.0009

WITTE H.H. (1988). Wave induced impact loading in deterministic and stochastic reflection. Mitt Leichtweiss Inst. Wasserbau, vol. 102, Tech. Univ. Braunschweig, Braunschweig, Germany.

WU G. (2007). Fluid impact on a solid boundary. Journal of fluids and structures, Vol. 23, pp 755-765. doi:10.1016/j.jfluidstructs.2006.11.002

YOUNGS D.L. (1982). Time-dependent multimaterial flow with large fluid distorsion. K.W. Morton and M.J. Baines, Numerical methods for fluids dynamics, Academic Press, New York.

YETTOU El-M., DESROCHERS A., CHAMPOUX Y. (2007). A new analytical model for pressure estimation of symmetrical water impact of a rigid wedge at variable velocities. Journal of fluids and structures Vol. 23, pp 501-522. 